\title{
Reduction of thermal conductivity in PbTe:Tl by alloying with $\mathrm{TlSbTe}_{2}$
}

\author{
Heng Wang, ${ }^{1}$ Anek Charoenphakdee, ${ }^{2}$ Ken Kurosaki, ${ }^{2}$ Shinsuke Yamanaka, ${ }^{2}$ and G. Jeffrey Snyder ${ }^{1, *}$ \\ ${ }^{1}$ Department of Materials Science, California Institute of Technology, Pasadena, California 91125, USA \\ ${ }^{2}$ Graduate School of Engineering, Osaka University, Suita, Osaka 565-0871, Japan
}

(Received 31 July 2010; published 27 January 2011)

\begin{abstract}
A series of single-phase polycrystalline $\left(\mathrm{TlSbTe}_{2}\right)_{x}\left(\mathrm{Tl}_{0.02} \mathrm{~Pb}_{0.98} \mathrm{Te}\right)_{1-x}(x=0,0.05,0.1)$ compounds were made to reduce thermal conductivity while maintaining the enhanced Seebeck coefficients found in PbTe doped with Tl. Transport property measurements confirmed that high Seebeck coefficients from doping with $\mathrm{Tl}$ are retained by alloying with $\mathrm{TlSbTe}_{2}$. At the same time, a thermal conductivity as low as $0.8 \mathrm{~W} / \mathrm{mK}$ at room temperature, and $0.6 \mathrm{~W} / \mathrm{mK}$ at $673 \mathrm{~K}$ was observed, corresponding to a $30 \%$ reduction in lattice thermal conductivity at $673 \mathrm{~K}$ compared with $2 \% \mathrm{Tl}-\mathrm{PbTe}$. However, the maximum $z T$ in this system is 0.8 (at $623 \mathrm{~K}$ ), which is lower than that of $2 \% \mathrm{Tl}-\mathrm{PbTe}$ prepared in this work $(1.2$ at $673 \mathrm{~K})$ owing to a decrease of the charge-carrier mobility when alloying with $\mathrm{TlSbTe}_{2}$. Possible influences on the mobility are discussed.
\end{abstract}

Direct conversion between heat and electricity via thermoelectricity is increasingly examined because of the demand for clean, efficient energy. Developing materials with high performance, i.e., high Seebeck coefficient $S$, low electric resistivity $\rho$, and low thermal conductivity $\kappa$, quantified by the thermoelectric figure of merit $z T=S^{2} T / \rho \kappa$, is of utmost importance for thermoelectric devices with satisfactory efficiency. Much progress has been made in this area over the past decade, ${ }^{1-4}$ particularly in PbTe-based systems, where dramatic increases of $z T$ have been observed. ${ }^{5-8}$ For example, $\mathrm{Tl}$ doping in PbTe increases the density of states near Fermi energy, resulting in high Seebeck coefficients coexisting with a high carrier concentration. ${ }^{9,10}$

While the Seebeck coefficient benefits from Tl doping, the thermal conductivity of Tl-PbTe is still at the same level as conventional PbTe. Because a common strategy to increase $z T$ is through reduction of thermal conductivity, one would expect an even higher $z T$ in Tl-PbTe when its thermal conductivity is reduced.

A well-known example of thermal conductivity reduction in PbTe-based materials is $\mathrm{AgPb}_{m} \mathrm{SbTe}_{2+m}$ (LAST) and other relevant systems, ${ }^{5-7,11}$ where nanometer-sized inclusions ${ }^{12,13}$ are thought to play a key role in scattering phonons while not affecting electrons.

Although effective in LAST, alloying with $\mathrm{AgSbTe}_{2}$ (or codoping of $\mathrm{Ag}$ and $\mathrm{Sb}$ ) is not an option for the Tl-PbTe system. Ag doping tends to replace or extract $\mathrm{Tl}$ from PbTe, which can be better understood given the fact that there are many stable ternary compounds such as AgTlTe (Ref. 14) and $\mathrm{Ag}_{9} \mathrm{TlTe}_{5}$ (Ref. 15) according to the phase diagram. Also, $\mathrm{AgSbTe}_{2}$ tends to provide electrons to PbTe, making all LAST materials $n$ type and thus will counteract $p$-type Tl-doping.

In this paper we report a strong reduction in thermal conductivity by alloying $\mathrm{Tl}_{0.02} \mathrm{~Pb}_{0.98} \mathrm{Te}$ (denoted here as $2 \% \mathrm{Tl}-\mathrm{PbTe}$ ) with $\mathrm{TlSbTe}_{2}$. Stoichiometric $\mathrm{TlSbTe}_{2}$ is a semiconductor (typically $p$ type) and stable up to $480^{\circ} \mathrm{C}$ with low thermal conductivity. ${ }^{16}$ Valence-balanced $\mathrm{TlSbTe}_{2}$ is unlikely to affect dramatically the doping of Tl-PbTe whether it forms a solid solution or a secondary phase. The rhombohedral lattice of $\mathrm{TlSbTe}_{2}$ has its close-packing plane of Te along (111) with a Te-Te distance of $4.42 \AA$. This is quite close to that in (111) of PbTe (4.52 $\AA$ ), thus providing the possibility of forming coherent precipitates in PbTe (111), leading to low thermal conductivities, although such precipitates are not directly observed in this paper.

Samples were made by using $\mathrm{TlSbTe}_{2}$ and $\mathrm{Tl}_{0.02} \mathrm{~Pb}_{0.98} \mathrm{Te}$ as the starting materials, each of which was first synthesized from the elements (with purities above 99.999\%). The materials were weighed according to nominal compositions of $\left(\mathrm{TlSbTe}_{2}\right)_{x}\left(\mathrm{Tl}_{0.02} \mathrm{~Pb}_{0.98} \mathrm{Te}\right)_{1-x}(x=0.05,0.1)$, then different processes were used. The first process involved solid-state diffusion, where the materials were mixed with a high-energy ball mill (SPEX 8000D) in steel vials for $30 \mathrm{~min}$, then cold pressed into pellets. The pellets were then sealed in quartz ampoules and annealed at $723 \mathrm{~K}$ for 28 days. After that the pellets were ground and hot pressed at $740 \mathrm{~K}$ for $1 \mathrm{~h}$, followed by annealing at $700 \mathrm{~K}$ for $90 \mathrm{~min}$ in vacuum. The second process involved a conventional melting technique: melting at $1250 \mathrm{~K}$ for $48 \mathrm{~h}$ and annealing at $800 \mathrm{~K}$ for 7 days, followed by the same sintering process. Samples are identified as TATL-95-d, TATL-90-d and TATL-95-m, TATL-90-m for each group. Here TATL is used for the system of $\left(\mathrm{TlSbTe}_{2}\right)_{x}\left(\mathrm{Tl}_{0.02} \mathrm{~Pb}_{0.98} \mathrm{Te}\right)_{1-x}$. The two digits stand for the percentage of $2 \% \mathrm{Tl}-\mathrm{PbTe}$ content following the nomenclature of "TAGS" (the compound with composition $\left(\mathrm{AgSbTe}_{2}\right)_{1-x}(\mathrm{GeTe})_{x}$ is usually marked as TAGS- $\left.x\right) .{ }^{17}$ The letter " $d$ " indicates the samples using solid-state diffusion and "m" refers to samples melted and annealed. Meanwhile, another group of sample was made following a third procedure: first melting $\mathrm{Tl}_{2} \mathrm{Te}$ with other elements at $1250 \mathrm{~K}$ for $48 \mathrm{~h}$ and then annealing at $800 \mathrm{~K}$ for 14 days, followed by hot pressing at $800 \mathrm{~K}$ for $3 \mathrm{~h}$ in $\mathrm{Ar}-\mathrm{H}_{2}$ atmosphere. They are identified as TATL-95-o, TATL-90-o as these were made at Osaka University, as described elsewhere. ${ }^{9}$

The resistivity and the Hall coefficient were measured by using the van der Pauw method. ${ }^{18}$ The mobility $(\mu=$ $\left.R_{H} / \rho\right)$ and Hall carrier density $\left(p=1 / R_{H} e\right)$ were calculated using a measured Hall coefficient. The thermal conductivity was calculated from the measured density $\rho$, the measured thermal diffusivity $\alpha$, and the calculated heat capacity $C_{p}$ by using a linear relationship $C_{p} / k_{B}$ atom $^{-1}=3.07+4.7 \times$ $10^{-4} \times(\mathrm{T} / \mathrm{K}-300)$, which, within $2 \%$ uncertainty, matches well with both reported $C_{p}$ for PbTe from measurements ${ }^{19}$ 

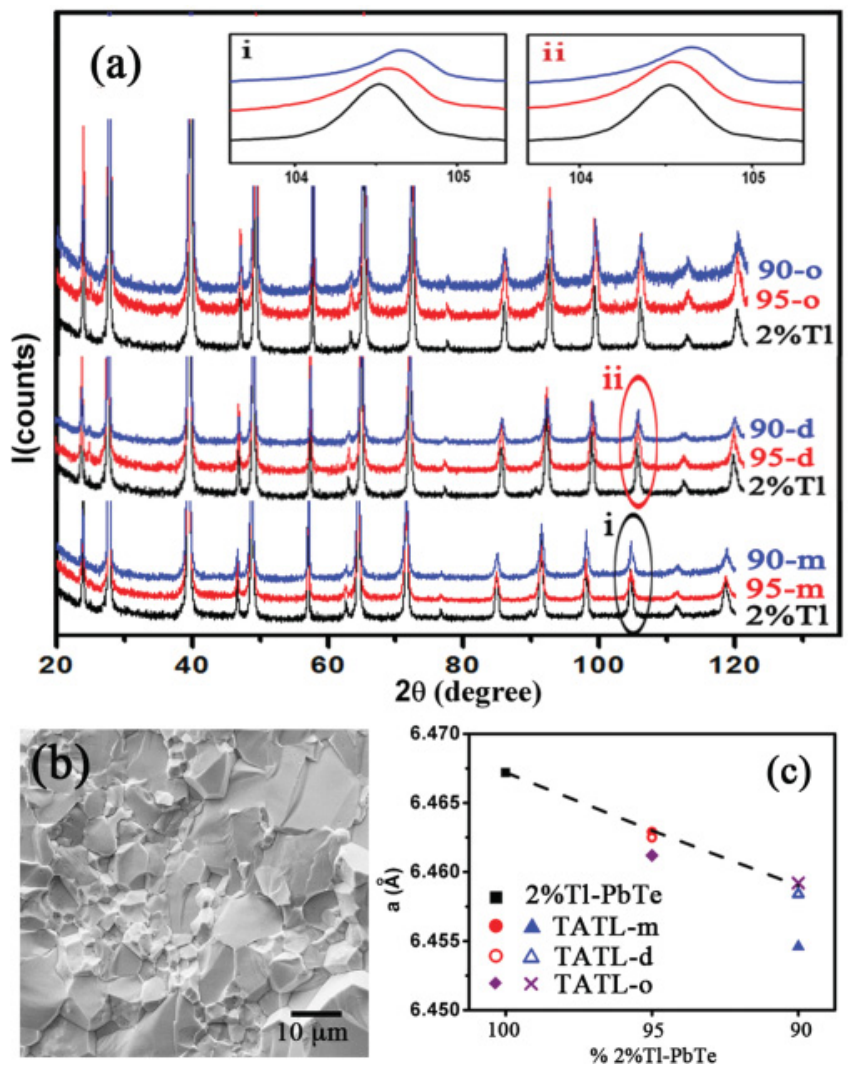

FIG. 1. (Color online) (a) XRD of samples with different $\mathrm{TlSbTe}_{2}$ content. (b) A typical fracture surface SEM image of the TATL sample. (c) The calculated lattice parameters.

and our calculation taking into account the Debye model and the contributions $s^{20}$ from lattice dilation and charge carriers. The Seebeck coefficient was measured using the slope of thermal voltage versus the temperature difference measured from fine $\mathrm{Nb}$-chromel thermocouples pressed onto the sample with a constant spring force during the measurement. The reported absolute Seebeck coefficient includes a small correction owing to the Seebeck voltage from the $\mathrm{Nb}$ wires.

All as-sintered samples have a geometric density above 97\% of the theoretical density for pure PbTe. All samples appear to have a single-phase fcc, $\mathrm{NaCl}$ structure by powder x-ray diffraction (XRD) [Fig. 1(a)]. In high-angle XRD patterns, the peaks shift toward higher $2 \theta$ angles as the content of $\mathrm{TlSbTe}_{2}$ increases, indicating a decrease of the lattice parameter $a$. This trend is clearly shown in Fig. 1(c) from the lattice parameter calculated from the XRD patterns. The grains that can be identified from fracture surface scanning electron microscopy (SEM) images are on the order of 50 $\mu \mathrm{m}$ [Fig. 1(b)]. In addition, backscattered electron SEM mapping on polished surfaces showed no secondary phase inclusions down to the resolution for this method (typically $<100 \mathrm{~nm}$ ).

Figure 2(a) shows the Seebeck coefficient ( $p$-type) at $300 \mathrm{~K}$ versus the Hall carrier density. The solid curve is obtained using the Boltzmann equation based on a single parabolic band (SPB) model, using a constant effective mass of $0.35 m_{e}$ and assuming that the acoustic phonon dominates the scattering
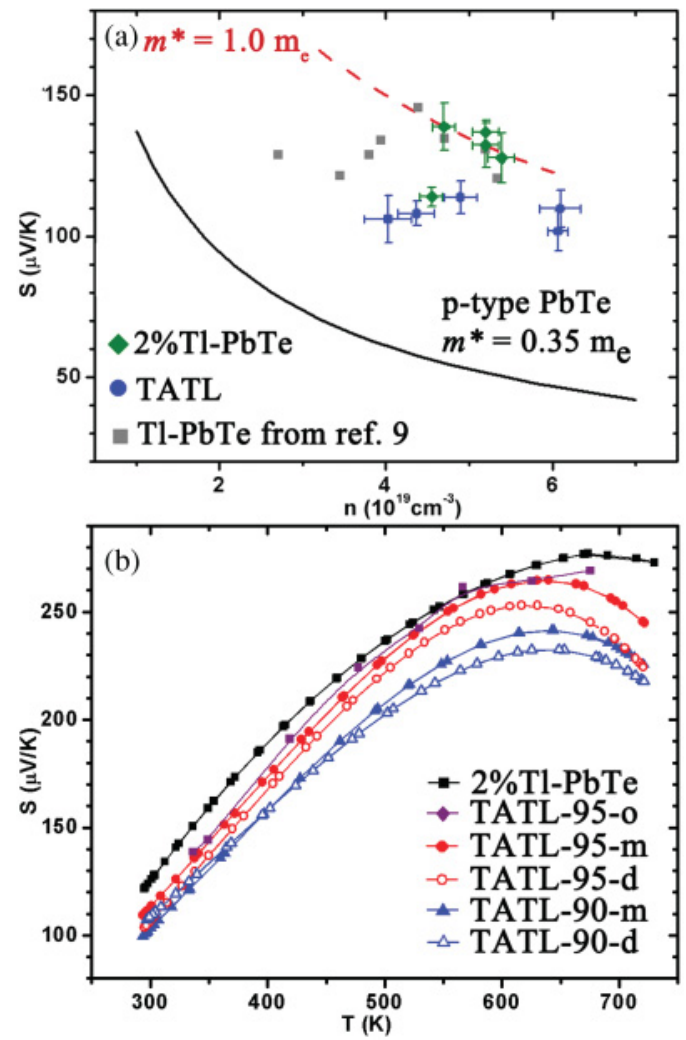

FIG. 2. (Color online) (a) Pisarenko plot of Tl-PbTe and TATL at room temperature. (b) Temperature dependence of the Seebeck coefficient for $2 \% \mathrm{Tl}-\mathrm{PbTe}$ and TATL.

$(r=0)$ :

$$
\begin{gathered}
n=\frac{4 \pi\left(2 m^{*} k_{B} T\right)^{3 / 2}}{h^{3}} F_{1 / 2}(\eta), \\
S=\frac{k_{B}}{e}\left[\frac{r+2}{r+1} \frac{F_{r+1}(\eta)}{F_{r}(\eta)}-\eta\right] .
\end{gathered}
$$

The Fermi integrals are defined as

$$
F_{n}(\eta)=\int_{0}^{\infty} \frac{x^{n}}{1+\exp (x-\eta)} d x .
$$

$m^{*}, \eta, h$, and $k_{B}$ are the effective mass, reduced chemical potential $\epsilon / k_{B} T$, Planck's constant, and the Boltzmann constant, respectively.

For a conventional $p$-type $\mathrm{PbTe}$, the $S$ - $p$ relationship is well described by the SPB curve $e^{21}$ up to $\sim 5 \times 10^{19} \mathrm{~cm}^{-3}$, while points for the Tl-PbTe system are found well above it: ${ }^{9}$ For $2 \% \mathrm{Tl}-\mathrm{PbTe}$ samples, an effective mass of $1 m_{e}$ would be required to fit the data using the SPB model. The Seebeck coefficient of TATL samples, represented by solid blue dots, are found slightly below those for their matrix $(2 \% \mathrm{Tl}-\mathrm{PbTe})$, which implies a slight decrease in effective mass; nevertheless, these points are still well above the conventional $p$-type $\mathrm{PbTe}$ curve, implying the Seebeck coefficient enhancement owing to a resonant level is maintained in TATL samples.

Figure 2(b) compares the Seebeck coefficients $S$ as a function of temperature. All TATL samples have a slightly lower $S$ compared with $2 \% \mathrm{Tl}-\mathrm{PbTe}$ at all temperatures. As more $\mathrm{TlSbTe}_{2}$ is added, the $S$ in the TATL samples decreases, 


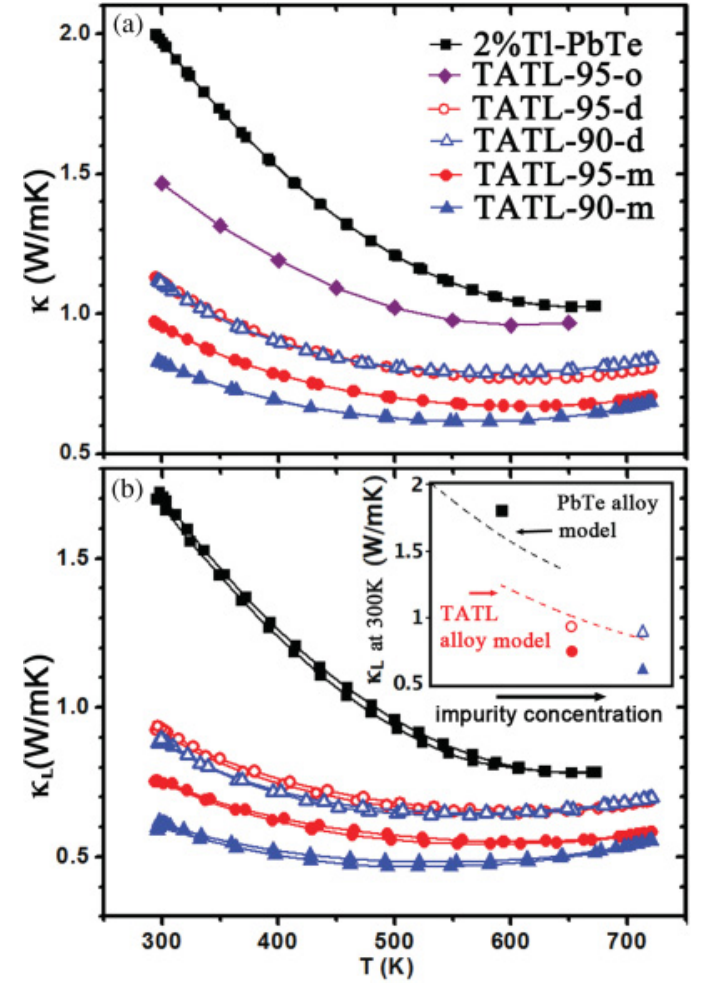

FIG. 3. (Color online) (a) Thermal conductivity and (b) its lattice component above room temperature up to $723 \mathrm{~K}$. Inset of (b) shows the estimated thermal conductivity assuming an alloy scattering effect (dashed line) as compared to measured results.

which can be expected because the carrier density is slightly increased [see the inset of Fig. 4(a)]. Also, the $S$ of all TATL samples begins to decrease at $\sim 650 \mathrm{~K}$, owing to the activation of minority carriers. Using the relation ${ }^{22} E_{g}=$ $2 e S_{\max } T_{\max }$, the slightly different peak in the $S$-vs- $T$ curves indicates a reduction in the effective band gap $\left(E_{g}\right)$ from $0.38 \mathrm{eV}$ for $2 \% \mathrm{Tl}-\mathrm{PbTe}$ to $0.31 \mathrm{eV}$ for TATL-90-m as more $\mathrm{TlSbTe}_{2}$ is added, which is consistent with the band-structure calculation ${ }^{23}$ of $\mathrm{TlSbTe}_{2}$.

Figure 3 shows the thermal conductivity for all samples. Compared with $2 \% \mathrm{Tl}-\mathrm{PbTe}$, a remarkable $50 \%$ reduction at room temperature can be seen in TATL samples. The lattice thermal conductivity $\kappa_{L}$ is obtained by subtracting the electronic contribution by using the Wiedemann-Franz Law, where the Lorenz number $L$ is calculated from the following expression for a single parabolic band:

$L=\left(\frac{k_{B}}{e}\right)^{2}\left\{\frac{r+3}{r+1} \frac{F_{r+2}(\eta)}{F_{r}(\eta)}-\left(\frac{r+2}{r+1}\right)^{2}\left[\frac{F_{r+1}(\eta)}{F_{r}(\eta)}\right]^{2}\right\}$.

The $L$ obtained for $2 \% \mathrm{Tl}-\mathrm{PbTe}$ is 1.81 at room temperature and 1.54 at $673 \mathrm{~K}$, while the $L$ for TATL samples are $\sim 1.88$ at room temperature and 1.57 at $673 \mathrm{~K}$.

As shown in Fig. 3(b), $\kappa_{L}$ at room temperature is reduced from $1.7 \mathrm{~W} / \mathrm{mK}$ for $2 \%$ Tl-PbTe to $0.9 \mathrm{~W} / \mathrm{mK}$ (TATL-95-d). Even at $673 \mathrm{~K}$ there is still a noticeable difference between $0.8 \mathrm{~W} / \mathrm{mK}$ for $2 \% \mathrm{Tl}-\mathrm{PbTe}$ and a minimum of $0.6 \mathrm{~W} / \mathrm{mK}$ for TATL samples.
Low thermal conductivities are often observed in solid solutions where point defects enhance phonon scattering owing to both mass and strain fluctuations. In such a disordered system, Callaway's model is used to describe the thermal conductivity governed by different scattering mechanisms. ${ }^{24}$ Specifically, if $T>\Theta_{D}$ and there is only umklapp and point-defect scattering, there is a simplified relation to predict $\kappa_{L}$ :

$$
\frac{\kappa_{L}}{\kappa_{L}^{P}}=\frac{\tan ^{-1} \omega}{\omega}, \omega=\sqrt{\frac{\pi^{2} \Theta_{D} \Omega}{h v^{2}} \kappa_{L}^{P} \Gamma} .
$$

Here $\kappa_{L}, \kappa_{L}^{P}, \Theta_{D}, \Omega, v$, and $\Gamma$ represents the lattice thermal conductivity of the disordered system, the lattice thermal conductivity of the ordered system before alloying, the Debye temperature, the volume per atom, the sound velocity, and the experimental disorder scattering parameter, respectively. We then consider the interpretation of $\Gamma$ proposed by Alekseeva et al. $:^{25,26}$

$$
\Gamma=\Gamma_{M}+\Gamma_{S},
$$

where $\Gamma_{M}$ and $\Gamma_{S}$ are contributions from the difference in mass and strain field. They are calculated using

$$
\begin{gathered}
\Gamma_{M}=\frac{1}{2}\left(\frac{\overline{M_{\mathrm{Pb}}}}{\overline{\bar{M}}}\right)^{2} \Gamma_{M}^{\mathrm{Pb}}+\frac{1}{2}\left(\frac{\overline{M_{\mathrm{Te}}}}{\overline{\bar{M}}}\right)^{2} \Gamma_{M}^{\mathrm{Te}}, \\
\Gamma_{S}=\frac{\xi}{2}\left(\frac{\overline{r_{\mathrm{Pb}}}}{\overline{\bar{r}}}\right)^{2} \Gamma_{S}^{\mathrm{Pb}}+\frac{\xi}{2}\left(\frac{\overline{r_{\mathrm{Te}}}}{\overline{\bar{r}}}\right)^{2} \Gamma_{S}^{\mathrm{Te}}, \\
\overline{M_{\mathrm{Pb}}}=f_{\mathrm{Tl}} M_{\mathrm{Tl}}+f_{\mathrm{Sb}} M_{\mathrm{Sb}}+f_{\mathrm{Pb}} M_{\mathrm{Pb}}, \\
\overline{\bar{M}}=\frac{1}{2} \overline{M_{\mathrm{Pb}}}+\frac{1}{2} \overline{M_{\mathrm{Te}}}=\frac{1}{2} \overline{M_{\mathrm{Pb}}}+\frac{1}{2} M_{\mathrm{Te}}, \\
\overline{r_{\mathrm{Pb}}}=f_{\mathrm{Tl}} r_{\mathrm{Tl}}+f_{\mathrm{Sb}} r_{\mathrm{Sb}}+f_{\mathrm{Pb}} r_{\mathrm{Pb}}, \\
\overline{\bar{r}}=\frac{1}{2} \overline{r_{\mathrm{Pb}}}+\frac{1}{2} \overline{r_{\mathrm{Te}}}=\frac{1}{2} \overline{r_{\mathrm{Pb}}}+\frac{1}{2} r_{\mathrm{Te}} .
\end{gathered}
$$

$M$ and $r$ are the atomic mass and the atomic radii for each element, and $\xi$ is an adjustable parameter related to the Grüneisen parameter; in this work we use $\xi=65$ as described in Ref. 25. The mass and strain fluctuation scattering parameters for the $\mathrm{Pb}$ sublattice are

$$
\begin{aligned}
\Gamma_{M}^{\mathrm{Pb}}= & f_{\mathrm{Tl}}\left(1-\frac{M_{\mathrm{Tl}}}{\overline{M_{\mathrm{Pb}}}}\right)^{2}+f_{\mathrm{Sb}}\left(1-\frac{M_{\mathrm{Sb}}}{\overline{M_{\mathrm{Pb}}}}\right)^{2} \\
& +f_{\mathrm{Pb}}\left(1-\frac{M_{\mathrm{Pb}}}{\overline{M_{\mathrm{Pb}}}}\right)^{2}, \\
\Gamma_{S}^{\mathrm{Pb}} & =f_{\mathrm{Tl}}\left(1-\frac{r_{\mathrm{Tl}}}{\overline{r_{\mathrm{Pb}}}}\right)^{2}+f_{\mathrm{Sb}}\left(1-\frac{r_{\mathrm{Sb}}}{\overline{r_{\mathrm{Pb}}}}\right)^{2} \\
& +f_{\mathrm{Pb}}\left(1-\frac{r_{\mathrm{Pb}}}{\overline{r_{\mathrm{Pb}}}}\right)^{2},
\end{aligned}
$$

whereas $\Gamma_{M}^{\mathrm{Te}}, \Gamma_{S}^{\mathrm{Te}}$ are zero because there is only one kind of atom on the Te site.

We used pure $\mathrm{PbTe}$ as the ordered system with ${ }^{21} \kappa_{L}=$ $2 \mathrm{~W} / \mathrm{mK}$. The room-temperature lattice thermal conductivity 
TABLE I. Thermal transport parameters used to estimate the alloy model for $\kappa$ in Tl-PbTe and TATL. The values are from speed of sound and XRD measurements on sintered polycrystalline samples. In all samples the contribution from strain field fluctuation $\Gamma_{S}$ overwhelms that from mass difference $\Gamma_{M}$.

\begin{tabular}{lccc}
\hline \hline & $2 \%$ Tl-PbTe & TATL-95 & TATL-90 \\
\hline$\xi$ & 65 & 65 & 65 \\
$M$ & 334.7 & 330.5 & 326.7 \\
$V_{l}(\mathrm{~m} / \mathrm{s})$ & 2900 & 2901 & 2850 \\
$V_{T}(\mathrm{~m} / \mathrm{s})$ & 1603 & 1612 & 1562 \\
$\Theta_{D}(\mathrm{~K})$ & 164 & 165 & 161 \\
$a(\AA)$ & 6.467 & 6.462 & 6.457 \\
$\Gamma_{M}$ & $2.8 \times 10^{-6}$ & 0.006 & 0.011 \\
$\Gamma_{S}$ & 0.018 & 0.107 & 0.188 \\
\hline
\end{tabular}

in the alloy model for Tl-PbTe and the TATL system is calculated [dashed line in the inset of Fig. 3(b)]. The result successfully explains $\kappa_{L}$ measured for $2 \% \mathrm{Tl}-\mathrm{PbTe}$ (parameters used are listed in Table I). While in TATL, the thermal conductivity reduction cannot be entirely explained by the alloy effect. This means there must be other mechanisms contributing to phonon scattering.

Figure 4(a) shows the mobility $\left(\mu=R_{H} / \rho\right)$ and carrier density $\left(p=1 / R_{H} e\right)$ from Hall measurements. All samples are heavily doped, with carrier densities on the order of $n \times$ $10^{19} \mathrm{~cm}^{-3} ; n$ changes from 4.4 to 6.1 among different TATL samples. In comparison, when measuring different $2 \% \mathrm{Tl}-\mathrm{PbTe}$
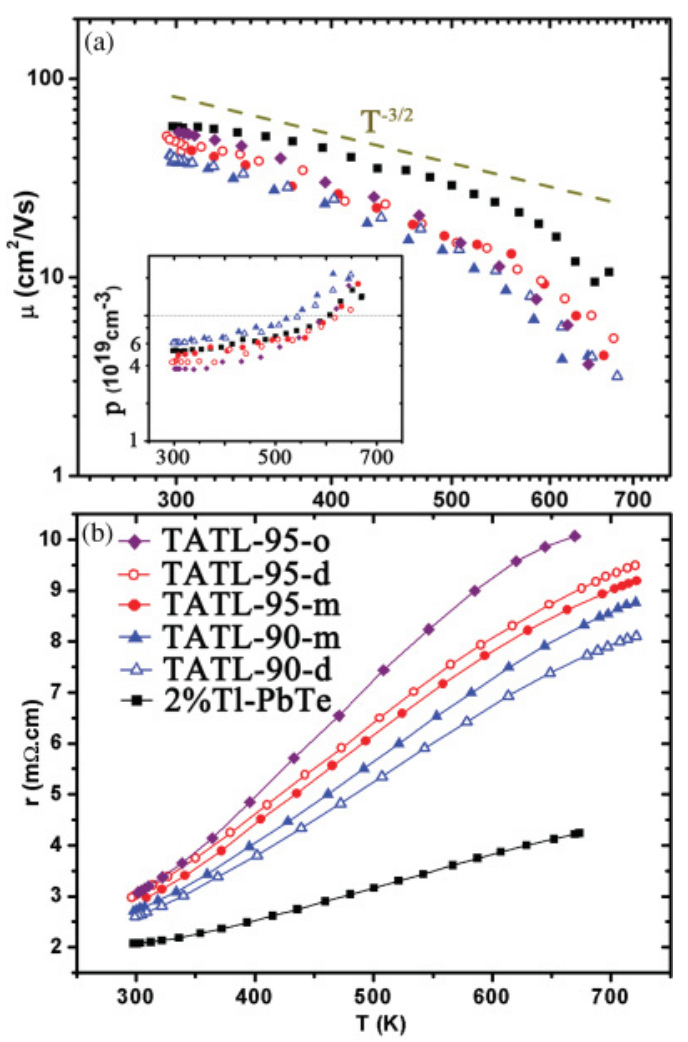

FIG. 4. (Color online) Temperature dependence of (a) mobility, carrier density [inset of (a)], and (b) resistivity of 2\% Tl-PbTe and TATL samples. samples, the result usually fluctuates from 4.5 to 5.5 . This indicates that $\mathrm{TlSbTe}_{2}$ does not considerably alter the doping level in the Tl-PbTe matrix.

Although the carrier densities are quite similar, all TATL samples differ from $2 \% \mathrm{Tl}-\mathrm{PbTe}$ in their mobility, which at room temperature is equal to $63 \mathrm{~cm}^{2} / \mathrm{Vs}$ for $2 \%$ Tl-PbTe but only $\sim 45 \mathrm{~cm}^{2} /$ Vs for TATL. As the temperature increases, they both decrease following the same relation $\mu \sim T^{-3 / 2}$ (data above $600 \mathrm{~K}$ are influenced by thermally excited minority carriers and are not considered here). This power law is expected for electron-acoustic-phonon dominant scattering and therefore we are led to assume electron-acoustic phonon interactions dominate the electron scattering in this temperature range. Other mechanisms such as grain-boundary scattering and impurity scattering are also expected to reduce the mobility. Nevertheless, because of the dominant effect of electron-acoustic-phonon scattering, it is important to consider it further. For an acoustic-phonon scattering dominant process, the mobility is given by ${ }^{21}$

$$
\mu=\frac{2^{3 / 2} \pi^{1 / 2} h^{4} e C_{l}}{(2 \pi)^{4} 3 m_{\chi}^{*} m_{d}^{* 3 / 2}\left(k_{B} T\right)^{3 / 2} \Xi^{2}} .
$$

Here $\mu$ is influenced by the following parameters: the conductivity effective mass and density-of-states effective mass, which are described by

$$
\frac{3}{m_{\chi}^{*}}=\frac{2}{m_{\perp}^{*}}+\frac{1}{m_{\|}^{*}} ; \quad m_{d}^{*}=\left(m_{\perp}^{*} m_{\|}^{* 2}\right)^{1 / 3} .
$$

The factor $C_{l}$ is determined by a combination of elastic moduli, which is further governed by the sound velocity, the molar mass, and the lattice constant. The factor $\Xi$ is related to a deformation potential and electron-phonon coupling. Note that there are other forms for this expression of $\mu$ that lead to related parameters. $^{27,28}$

The sound velocities of TATL were measured and found to be virtually identical to $2 \% \mathrm{Tl}-\mathrm{PbTe}$, along with the molar mass and lattice constants (Table I). The smaller effective mass of TATL, as revealed by the Pisarenko plot [Fig. 2(a)], should enhance the mobility as opposed to the observed decrease compared to $2 \% \mathrm{Tl}-\mathrm{PbTe}$. Thus, assuming the mobility is dominated by acoustic-phonon scattering, the lower mobility in TATL should be attributed to a larger $\Xi$ factor, i.e., a

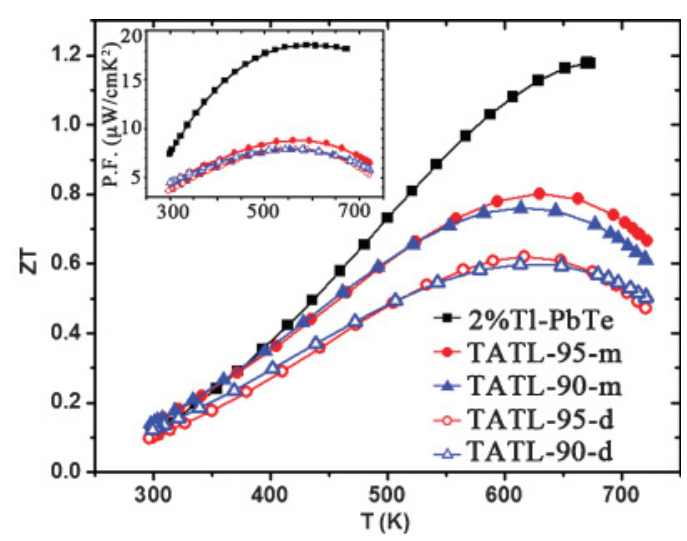

FIG. 5. (Color online) Temperature dependence of power factor (inset) and $z T$ of TATL as compared to $2 \% \mathrm{Tl}-\mathrm{PbTe}$. 
stronger interaction between electrons and phonons, which would include all the terms related to the electron-phonon interaction, including the phonon density of states.

The temperature-dependent resistivity [Fig. 4(b)] shows the dramatic effect of the reduced mobility of TATL. TATL samples have a $50 \%$ higher resistivity at room temperature as compared to $2 \% \mathrm{Tl}-\mathrm{PbTe}$, and at $673 \mathrm{~K}$ this difference is further increased to $>100 \%$. The $1 \% \mathrm{Tl}-\mathrm{PbTe}$ has a similar temperature dependence $^{9}$ as observed in TATL, but this is owing to its lower carrier density $\left(\sim 2.5 \times 10^{19} \mathrm{~cm}^{-3}\right)$ and not to a lower mobility.

In total, although the thermal conductivity in TATL is effectively reduced, the benefit to $z T$ is canceled out by the increase of resistivity. As shown in the inset of Fig. 5, the power factor is considerably lower in TATL as compared to $2 \% \mathrm{Tl}-\mathrm{PbTe}$. The $z T$ in the TATL system reaches a maximum of 0.8 at $623 \mathrm{~K}$ for TATL-95-m, in contrast to $z T=1.2$ for $2 \% \mathrm{Tl}-\mathrm{PbTe}$ prepared and measured in the same manner at 673 K (Fig. 5).
It is possible that nanostructures may improve $z T$ in Tl-PbTe, but careful tuning of carrier densityas well as the microstructure and even electron-phonon interaction may be required. Otherwise, as in the case of TATL, the power factor is likely to be reduced seriously enough to compensate the benefits brought by $\kappa$ reduction, resulting in a reduced $z T$.

In summary, polycrystalline $\left(\mathrm{TlSbTe}_{2}\right)_{x}\left(\mathrm{Tl}_{0.02} \mathrm{~Pb}_{0.98} \mathrm{Te}\right)_{1-x}$ alloys with $x=0,0.05,0.1$ were made and a thermal conductivity reduction of up to $30 \%$ compared to $2 \% \mathrm{Tl}-\mathrm{PbTe}$ at $673 \mathrm{~K}$ was observed. The results show that the Tl-PbTe system can maintain its enhanced Seebeck coefficient while its thermal conductivity is reduced when alloyed. However, for TATL, the mobility is also substantially decreased, leading to lower $z T$. In order to improve the overall performance of $\mathrm{Tl}-\mathrm{PbTe}$ with alloying, the impact on electron mobility will need to be controlled.

This work is supported by BSST/ZTPlus and NASA-Jet Propulsion Laboratory.
*Corresponding author: jsnyder@ caltech.edu.

${ }^{1}$ G. J. Snyder and E. S. Toberer, Nat. Mater. 7, 105 (2008).

${ }^{2}$ A. J. Minnich, M. S. Dresselhaus, Z. F. Ren, and G. Chen, Energy Environ. Sci. 2, 466 (2009).

${ }^{3}$ J. R. Sootsman, D. Y. Chung, and M. G. Kanatzidis, Angew. Chem. Int. Ed. 48, 8616 (2009).

${ }^{4}$ D. L. Medlin, and G. J. Snyder, Curr. Opin. Colloid Interface Sci. 14, 226 (2009).

${ }^{5}$ K. F. Hsu, S. Loo, F. Guo, W. Chen, J. S. Dyck, C. Uher, T. Hogan, E. K. Polychroniadis, and M. G. Kanatzidis, Science 303, 818 (2004).

${ }^{6}$ J. Androulakis, K. F. Hsu, R. Pcionek, H. Kong, C. Uher, J. J. D’Angelo, A. Downey, T. Hogan, and M. G. Kanatzidis, Adv. Mater. (Weinheim, Ger.) 18, 1170 (2006).

${ }^{7}$ P. F. P. Poudeu, J. D'Angelo, A. D. Downey, J. L. Short, T. P. Hogan, and M. G. Kanatzidis, Angew. Chem. Int. Ed. 45, 3835 (2006).

${ }^{8}$ J. R. Sootsman, H. Kong, C. Uher, J. J. D'Angelo, C. I. Wu, T. P. Hogan, T. Caillat, and M. G. Kanatzidis, Angew. Chem. Int. Ed. 47, 8618 (2008).

${ }^{9}$ J. P. Heremans, V. Jovovic, E. S. Toberer, A. Saramat, K. Kurosaki, A. Charoenphakdee, S. Yamanaka, and G. J. Snyder, Science 321, 554 (2008).

${ }^{10}$ S. A. Nemov and Y. I. Ravich, Phys. Usp. 41, 735 (1998).

${ }^{11}$ P. F. P. Poudeu, J. D’Angelo, H. Kong, A. Downey, J. L. Short, R. Pcionek, T. P. Hogan, C. Uher, and M. G. Kanatzidis, J. Am. Chem. Soc. 128, 14347 (2006).

${ }^{12}$ E. Quarez, K. F. Hsu, R. Pcionek, N. Frangis, E. K. Polychroniadis, and M. G. Kanatsidis, J. Am. Chem. Soc. 127, 9177 (2005).

${ }^{13}$ L. Wu, J. C. Zheng, J. Zhou, Q. Li, J. Yang, and Yimei Zhu, J. Appl. Phys. 105, 094317 (2009).
${ }^{14}$ K. Kurosaki, K. Goto, H. Muta, and S. Yamanaka, J. Appl. Phys. 102, 023707 (2007).

${ }^{15}$ K. Kurosaki, A. Kosuga, H. Muta, M. Uno, and S. Yamanaka, Appl. Phys. Lett. 87, 061919 (2005).

${ }^{16} \mathrm{~K}$. Kurosaki, H. Uneda, H. Muta, and S. Yamanaka, J. Alloys Compd. 376, 43 (2004).

${ }^{17}$ E. A. Skrabek and D. S. Trimmer, in CRC Handbook of Thermoelectrics, edited by D. M. Rowe (CRC, Boca Raton, FL, 1995), pp. 267-275.

${ }^{18}$ I. A. Nishida, in CRC Handbook of Thermoelectrics, edited by D. M. Rowe (CRC, Boca Raton, FL, 1995), pp. 158-160.

${ }^{19}$ R. Blachnik and R. Igel, Z. Naturforsch. B 29b, 625 (1974).

${ }^{20}$ O. Delaire, A. F. May, M. A. McGuire, W. D. Porter, M. S. Lucas, M. B. Stone, D. L. Abernathy, V. A. Ravi, S. A. Firdosy, and G. J. Snyder, Phys. Rev. B 80, 184302 (2009).

${ }^{21}$ Y. I. Ravich and U. Isaakovich, Semiconducting Lead Chalcogenides (Plenum, New York, 1970), pp. 90, 157, and 209.

${ }^{22}$ H. J. Goldsmid and J. W. Sharp, J. Electron. Mater. 28, 869 (1999).

${ }^{23}$ K. Hoang and S. D. Mahanti, Phys. Rev. B 77, 205107 (2008).

${ }^{24}$ J. Callaway and H. C. Von Baeyer, Phys. Rev. 120, 1149 (1960).

${ }^{25}$ G. T. Alekseeva, B. A. Efimova, L. M. Ostrovskaya, O. S. Serebryannikova, and M. I. Tsypin, Sov. Phys. Semicond.-USSR 4, 1122 (1971).

${ }^{26}$ J. Yang, G. P. Meisner, and L. Chen, Appl. Phys. Lett. 85, 1140 (2004).

${ }^{27}$ E. S. Toberer, C. A. Cox, S. R. Brown, T. Ikeda, A. F. May, S. M. Kauzlarich, and G. J. Snyder, Adv. Funct. Mater. 18, 2795 (2008).

${ }^{28}$ S. Ahmad, and S. D. Mahanti, Phys. Rev. B 81, 165203 (2010). 